carotid elasticity and is an efficient method to evaluate artery elasticity.

\section{GW23-e1308 ASSESSMENT OF CAROTID ARTERY ELASTICITY IN THE NORMAL POPULATION BY TWO-DIMENSIONAL SPECKLE TRACKING IMAGING}

doi:10.1136/heartjnl-2012-302920z.5
Yao Deng, Mingxing Xie. Department of Ultrasonography, Union Hospital of Tongji Medical College, Huazhong University of Science and Technology; Hubei Provincial Key Laboratory of Molecular Imaging, Wuhan 430022, China

Objectives To investigate the value of two-dimensional speckle tracking imaging (2D-STI) in the assessment of carotid circumferential strain of the normal population.

Methods Totally 140 healthy people (age 20-95 years old), according to age, divided into seven groups (the 1 group: 20 to 29, the 2 group: 30 to 39 , the 3 group: 40 to 49 , the 4 group: 50 to 59 , the 5 group: 60 to 69 , the 6 group: 70 to 79 , the 7 group: older than 79 , each group male and female, respectively 10). High frame rate dynamic $2 \mathrm{D}$ images were recorded from short axis view at the left common carotid artery below the sinus $1.5-2.0 \mathrm{~cm}$ application of Esaote My Lab 90. The global peak circumferential strain ( $\square$ ) was measured with 2D-STI; the application OIMT and OAS measuring Carotid Intima-Media Thickness (IMT), distensibility coefficient (DC), compliance coefficient (CC), stiffness parameter $(\alpha, \beta)$ and pulse wave velocity (PWV $\beta)$. All parameters were compared between groups.

Results From the 1 group to 7 group $\square$, DC, CC decreases gradually, while the IMT, $\alpha, \beta$, PWV $\beta$ gradually increase, however, the trends of the 5 group to 7 group are slow. The comparison between the 1 group and the 3 group to the 7 group all parameters differences were statistically significant $(p<0.05)$, The comparison between the 2 group and the 3 group to the 7 group all parameters differences were statistically significant $(p<0.05)$, the comparison between the 3 group and the 5 group to the 7 group part of the parameters differences were statistically significant $(p<0.05)$, The comparison between the 4 group and the 6 group to the 7 group part of parameters differences were statistically significant $(p<0.05)$, The comparison between the 5 group and the 6 group, the 7 group only IMT differences were statistically significant $(p<0.05)$, compared with the values of the 6 group and the 7 group, there was no significant difference ( $\mathrm{p}>0.05)$.

Conclusions The carotid artery $\square$, IMT, the elastic parameters of the healthy population increased with age, 2D-STI can observe 